\title{
The Relationship Between Hip Strength and Postural Stability in Collegiate Athletes Who Participate in Lower Extremity Dominant Sports
}

Bryce Olsen ${ }^{1}$, Nicholas Freijomil', Jennifer Csonka, MS, LAT, ATC ${ }^{2}$, Tara Moore, MS, LAT, ATC ${ }^{3}$, Carolyn Killelea, PhD ${ }^{4}$ Mallory S Faherty, PhD, LAT, ATC ${ }^{4}$, Timothy C Sell, PhD, PT ${ }^{4}$

1 Division of Physical Therapy, Department of Orthopedics, Duke University, ${ }^{2}$ Department of Athletics, University of Pittsburgh, ${ }^{3}$ Department of Athletic Medicine, Duke University, ${ }^{4}$ Michael W. Krzyzewski Human Performance Laboratory, Department of Orthopaedic Surgery, Duke University Keywords: rehabilitation, postural stability, physical therapy, movement system, injury prevention, hip strength, balance https://doi.org/10.26603/001c.18817

\section{International Journal of Sports Physical Therapy}

\section{Background}

Lower extremity (LE) injuries are common across many sports. Both core strength (including hip strength) deficits and poor postural stability have been linked to lower extremity (LE) injury. The relationship between these two characteristics is unknown.

\section{Purpose}

To explore the relationships between hip strength, static postural stability, and dynamic postural stability.

\section{Study Design}

Descriptive Cross-Sectional Study

\section{Methods}

162 Division I student-athletes (111 males and 51 females) participated in this study. Isometric hip strength was measured using a hand-held dynamometer and both single-leg static (eyes open EO and eyes closed EC) and dynamic postural stability were assessed with a force plate. Pairwise correlations were calculated to examine the relationship between the hip strength variables and the postural stability scores for all subjects and separately for males and females.

\section{Results}

There were no significant correlations between hip strength and dynamic postural stability for any of the pairwise correlations. Significant, albeit minimal, correlations between EO and EC static postural stability and each of the hip strength variables for all subjects and male subjects (correlation coefficients ranged from -0.19 to -0.34 ). However, there were only two significant correlations between hip strength and EC static postural stability (hip internal/external rotation) and one for hip strength and EO postural stability (hip internal rotation) found for female subjects (correlation coefficients ranged from -0.28 to -0.31$)$.

\section{Conclusion}

There was no relationship between isometric hip strength and dynamic postural stability; whereas, there were some relationships between the strength measures and static

\footnotetext{
a Corresponding Author:

Bryce Olsen

Duke University, Division of Physical Therapy

Department of Orthopedics

Durham, NC, 27708

801-400-5368

Bryce.j.olsen.17@gmail.com
} 
Table 1: Subject Demographics

\begin{tabular}{|c|c|c|c|c|c|c|}
\hline & \multicolumn{3}{|l|}{ Males } & \multicolumn{3}{|l|}{ Females } \\
\hline & $\mathrm{n}$ & Mean & $\pm \mathrm{SD}$ & $\mathrm{n}$ & Mean & $\pm \mathrm{SD}$ \\
\hline Age (years) & 111 & 19.6 & 1.4 & 51 & 19.3 & 1.2 \\
\hline Height $(\mathrm{cm})$ & 111 & 185.4 & 8.6 & 51 & 172.9 & 8.7 \\
\hline Weight (kg) & 111 & 84.9 & 14.1 & 51 & 67.5 & 10.1 \\
\hline
\end{tabular}

postural stability. These significant, but minimal correlations were observed in more of the comparisons within the male cohort potentially demonstrating a sex difference.

\section{Level of Evidence}

$3 \mathrm{~b}$

\section{INTRODUCTION}

A musculoskeletal injury is a common occurrence for nearly all NCAA student-athletes at some point during their collegiate career. NCAA injury surveillance data has reported the likelihood of an injury occurring to be as high as one injury every two games for a team of 50 participants. ${ }^{1}$ Injuries negatively affect the player including missed participation in sport, decreased level of performance upon return, and potential long-term health issues. ${ }^{2}$ Lower extremity (LE) injuries make up over half of all injuries experienced by NCAA athletes, with a majority of LE injuries involving the knee and ankle. ${ }^{1}$ A better understanding of the factors that lead to LE injuries will help guide and refine injury prevention programs for NCAA athletes.

In addition to previous injury, there are several other examples of identified risk factors for common lower extremity injuries in student-athlete populations. Reduced muscular strength and decreased neuromuscular control in the lower extremity have been identified as risk factors for knee injury. ${ }^{3}$ Increased tibial varum and calcaneal eversion in women and increased talar tilt in men have been shown to be predictive of ankle injury. 4 Two measures that are increasingly being researched in connection with LE injury risk are postural stability and core strength.

Postural stability is the ability to integrate sensory, motor, and vestibular input within the execution of motor commands in order to maintain stability and equilibrium in the midst of a variety of perturbations. ${ }^{5}$ Static and dynamic postural stability, as demonstrated through various tests of postural sway, were shown to be predictive of ankle injury. ${ }^{6,7}$ Core musculature refers to the abdominals, paraspinals, and the musculature surrounding the hip complex. ${ }^{8}$ Theories have pointed to deficits in core strength increasing the risk for LE injury potentially due to the inability to transfer energy through the core and thereby putting greater stress on the LE. ${ }^{8,9}$ For example, both decreased hip external rotation strength and poor core-muscle endurance have been proposed as risk factors for LE injury. ${ }^{10,11}$

Student-athletes frequently suffer lower extremity injuries demonstrating a continued need to develop injury prevention strategies to reduce their risk of injury. Core strength, including hip strength, and postural stability have both been linked to lower extremity injury; ${ }^{10,12}$ however, the relationship between hip strength and postural stability measures, especially dynamic postural stability, are not well known. The purpose of this study was to examine the relationship between hip strength and both static and dynamic postural stability. It was hypothesized that there would be significant correlations between isometric hip strength and dynamic postural stability and no significant correlations between isometric hip strength and static postural stability. The results of this study may guide future injury prevention programs and research examining injury prevention protocols for student-athletes.

\section{METHODS}

\section{EXPERIMENTAL DESIGN}

This study utilized a prospective cohort design. A descriptive cohort design was chosen in order to determine the association between hip strength and both static and dynamic postural stability in NCAA Division I male and female athletes.

\section{SUBJECTS}

A total of 162 Division I student-athletes (soccer, basketball, and football) from two different universities volunteered for the study. Subjects consisted of 111 males and 51 females. Demographic information for the subjects is presented in Table 1 . All subjects voluntarily consented to participate in the study and signed an informed consent approved by their university's Institutional Review Board. All subjects were currently cleared for full participation in their team's activities.

\section{INSTRUMENTATION}

A handheld dynamometer was utilized to assess isometric muscle strength (Lafayette Instrument Co., Lafayette, IN). For all measures, peak force was measured to the nearest 0.1 kilogram. A Kistler force plate (Kistler 9286A, Amherst, $\mathrm{NY}$ ) was utilized to collect ground reaction force data in order to assess static and dynamic postural stability. The force plate was calibrated according to manufacturer's rec- 
ommendations prior to the initiation of any testing procedures. A sampling frequency of $200 \mathrm{~Hz}$ and $1200 \mathrm{~Hz}$ was utilized for static postural stability and dynamic postural stability, respectively. All force plate data was passed through an amplifier followed by an analogy digital board and lastly stored on a personal computer. A custom MATLAB (MathWorks, v7.0.4, Natick, MA) script was utilized to process all ground reaction force data.

\section{PROCEDURES}

Dynamic postural stability was assessed during a single leg jump test. This protocol has been previously demonstrated to be reliable. ${ }^{13}$ Jump distance was normalized to body height (40\%). Subjects were required to complete a jump in the anterior direction over a $30 \mathrm{~cm}$ hurdle that was placed midway between the starting point and the front edge of the force plate. Subjects began the jump with a two-footed take-off over the hurdle and were instructed to land on the force plate on the tested leg, stabilize as quickly as possible, place hands on hips, and balance for five seconds while looking straight ahead. Trials were discarded and repeated if subjects failed to jump over or came in contact with the hurdle, hopped on the test leg after landing, the non-weight-bearing leg touched down, or if subjects removed their hands from hips for longer than three seconds. Subjects were provided with three practice trials prior to testing with a one-minute rest period between the practice and test trials. Testing was performed bilaterally. Five successful trials were collected and averaged on both lower extremities; however, data were only used from the dominant leg. Dominant leg was determined by asking participant which leg they would kick a ball with.

Single-leg static postural stability was assessed during a single-leg stance test. The test was performed barefooted under eyes-open and eyes-closed conditions. Subjects performed three trials lasting 10 seconds each for each condition. During testing, the subject was asked to keep the nontest lower extremity foot raised to mid-tibia of the tested lower extremity without touching the tested limb, while maintaining hands on hips. If the subject touched legs or touched down outside the force plate at any time, the trial was discarded and recollected. Data analysis was completed via the standard deviation of ground reaction forces as described later. This protocol has been previously demonstrated to be reliable. ${ }^{14}$ Subjects were provided one complete practice trial for each condition for each leg.

Isometric strength was tested using a handheld dynamometer. Peak force was measured with the dynamometer to the nearest $0.1 \mathrm{~kg}$ on both the dominant and the non-dominant lower extremity. This assessment of strength was a "make test", as the subject was asked to exert as much force as possible against an unmoving resistance (a researcher). A practice trial at 50\% of maximum effort was provided for each testing position in order to ensure proper performance. Subjects rested for 30 seconds between each trial in order to avoid fatigue. Three trials were measured, and the results were averaged. Hip abduction, adduction, internal rotation, and external rotation were tested according to standard strength testing positioning, as described below. ${ }^{15}$
Hip abduction strength was tested with the subject positioned side lying with the leg to be tested on top. The test leg was supported by a pillow under the lower limb in order to support a neutral spine throughout the duration of the test. The bottom leg was positioned in 90 degrees of knee flexion. The handheld dynamometer was placed just superior to the lateral malleolus. The subject was instructed to exert a maximal force against the handheld dynamometer while the examiner maintained a stationary position with the handheld dynamometer.

Hip adduction strength was tested with the subject positioned side lying with the leg to be tested on the bottom. The top leg was positioned in 90 degrees of knee flexion with a pillow at the knee in order to support a neutral spine throughout the duration of the test. The handheld dynamometer was placed just superior to the medial malleolus. The subject was instructed to exert a maximal force against the handheld dynamometer while the examiner maintained a stationary position with the handheld dynamometer.

Hip internal rotation strength was tested with the subject positioned prone on a treatment table with the knee of the test leg flexed to 90 degrees. The examiner passively positioned the test leg into a neutral hip internal-external rotation prior to the initiation of the test. The handheld dynamometer was placed just distal to the lateral malleolus. The subject was instructed to exert a maximal force against the handheld dynamometer while the examiner maintained a stationary position with the handheld dynamometer

Hip external rotation strength was tested with the subject positioned prone on a treatment table with the knee of the test leg flexed to 90 degrees. The examiner passively positioned the test leg into a neutral hip internal-external rotation prior to the initiation of the test. The handheld dynamometer was placed just distal to the medial malleolus. The subject was instructed to exert a maximal force against the handheld dynamometer while the examiner maintained a stationary position with the handheld dynamometer.

\section{STATISTICAL METHODS}

Data analysis was completed using the standard deviation of ground reaction forces in three planes (anterior/posterior, medial/lateral, and vertical) and the center of pressure in two planes (anterior/posterior and medial/lateral) during the 10 -second trial.

For all measures of isometric strength, peak force was averaged across three successful trials was captured in kilograms and normalized to body weight for comparison across subjects $(\% \mathrm{BW}=$ (average $(\mathrm{kg}) /$ subject body weight $\left.(\mathrm{kg}))^{*} 100\right)$. Isometric strength measurements normalized to body weight was utilized to calculate strength ratios between each subject's dominant and non-dominant lower extremity. Isometric strength measurements normalized to body weight was also utilized to calculate strength ratios between opposing muscle groups on each subject's dominant and non-dominant lower extremity which included hip adduction/abduction and hip external/internal rotation.

Static postural stability is expressed as the standard deviation of ground reaction forces. Following the completion of data collection, a custom Matlab (MathWorks, v7.0.4, 
Natick, MA) script was used to process and filter the data. All ground reaction force data were passed through a lowpass Butterworth filter with a cut off frequency of $20 \mathrm{~Hz}$. The ground reaction forces from each successful trial were normalized to body weight (\%BW). The standard deviation of the ground reaction forces in the anterior/posterior, medial/lateral, and vertical directions, as well as center of pressure in the anterior/posterior and medial/lateral directions were calculated across three successful trials for all conditions. An average of the ground reaction forces in each direction for each condition was calculated in order to explain the subject's overall static proprioception under each condition. For this protocol and data analysis, increased variability in movement on the force plates demonstrates decreased postural stability and a decreased ability to stick the landing at initial contact. Composite scores were calculated for static postural stability and averaged across three trials. In prior laboratory piloting and testing, three trials were demonstrated to be highly reliable for the static balance tasks (ICC $=0.759-0.879$; SEM $=0.187-1.616) .{ }^{16}$

Dynamic postural stability is expressed through the use of the Dynamic Postural Stability Index. The Dynamic Postural Stability Index calculation creates a stability index for each anatomical direction as well as a composite of all three directions utilizing the first three seconds of ground reaction force data following initial contact with the force plate. For the purposes of this study, initial contact is defined as the point in which the vertical ground reaction force exceeds five percent of the subject's body weight. Following the completion of data collection, a custom Matlab (MathWorks, v7.0.4, Natick, MA) script processes and filters the data. All ground reaction force data was passed through a low-pass Butterworth filter with a cut off frequency of 20 Hz. An individual Dynamic Postural Stability Index score was calculated for each of the five successful trials. An average of the Dynamic Postural Stability Index scores from the five successful trials was calculated in order to explain a subject's overall dynamic postural stability. The primary variable for the AP jump and ML jump was the Dynamic Postural Stability Index (DPSI). As described in regard to the static postural stability tasks, a higher DPSI also represents worse postural stability. Intersession reliability and standard errors of measurement were calculated for the primary measures during both postural stability tasks. An intraclass correlation (ICC) using the model described by Shrout and Fleiss was employed to determine the intersession reliability. ${ }^{17}$

$$
\text { DPSI }=((\sqrt{[} \Sigma(0-\mathrm{x}) 2+\Sigma(0-\mathrm{y}) 2+(\text { Body Weight }-z) 2 /
$$$$
\text { Number of Data Points])/Body Weight) }
$$

Statistical analysis was performed for all subjects and then again separately for males and females. An alpha level of 0.05 was set a priori to determine significance for all statistical analyses. A series of 12 bivariate correlations (Pearson's $r$ ) were computed to determine if a relationship existed between the static and dynamic postural stability measures and the strength measures.

\section{RESULTS}

Mean, standard deviation, min, and max values for hip strength and postural stability are listed in Table 2 broken down by males and females. Pairwise correlations, including $r$ coefficients and $\mathrm{p}$ values, between hip strength and postural stability measures are listed in Tables $\underline{3}, \underline{4}$, and $\underline{5}$ representing all subjects, male subjects, and female subjects, respectively. Among all subjects, significant, albeit weak, correlations were found between all hip strength measures (adduction, abduction, IR, ER) and static postural stability in both the eyes open and eyes closed conditions (correlation coefficients ranged from -0.19 to -0.29). Among male subjects, significant but weak correlations were found between all hip strength measures and static postural stability in both the eyes open and eyes closed conditions (correlation coefficients ranged from -0.21 to -0.34 ). Among female subjects, static postural stability with eyes closed condition found significant but weak correlations with internal and external rotation strength and static postural stability with eyes open condition found a significant correlation with internal rotation strength (correlation coefficients ranged from -0.28 to -0.31 ). All other static postural stability and hip strength correlations among females were not significant. Among all subjects, males, and females there were no significant correlations between dynamic postural stability and hip strength measures.

\section{DISCUSSION}

The main finding of this study was the presence of significant, albeit weak, correlations between isometric hip strength and static postural stability measures, and no correlation between isometric hip strength and dynamic postural stability measures. These findings refuted the original hypothesis of a correlation between dynamic postural stability measures and core strength and no correlation between static postural stability measures and core strength. The findings also revealed a sex difference which could be further examined in future studies. While some weak correlations were found, the relationship between isometric hip strength and postural stability is likely much more complicated than the factors assessed in the current study, and will require additional research.

The results of this study showed no association between isometric hip strength and dynamic postural stability across the entire group or within each sex. These results do not support the original hypothesis and differ from the results of other investigations. Ambegaonkar et al and Wilson et al both looked at the relationship between isometric hip strength and dynamic postural stability and found significant correlations between measurements of hip strength and dynamic balance scores. ${ }^{18,19}$ Both studies used similar methods to this study of using a handheld dynamometer for determining isometric hip strength, however this study utilized the Dynamic Postural Stability Index (DPSI) generated from a force plate during single leg landing activities to determine dynamic postural stability whereas Ambegaonkar et al utilized the Star Excursion Balance Test (SEBT) ${ }^{18}$ and Wilson et al utilized the Y Balance Test. ${ }^{19}$ The contradictory findings may be due to how postural stability was assessed.

The DPSI, SEBT, and Y-Balance Test are all considered tests of dynamic postural stability, but the demands each test places on postural stability likely are different. The 
Table 2: Hip Strength, Static Postural Stability*, and Dynamic Postural Stability**

\begin{tabular}{|c|c|c|c|c|c|c|c|c|}
\hline & \multicolumn{4}{|l|}{ Males } & \multicolumn{4}{|c|}{ Females } \\
\hline & Mean & $\pm \mathrm{SD}$ & $\min$ & $\max$ & Mean & $\pm \mathrm{SD}$ & $\min$ & $\max$ \\
\hline Hip Abduction (\% Bodyweight) & 19.3 & 5.2 & 10.5 & 37.2 & 20.03 & 4.9 & 10 & 33.3 \\
\hline Hip Adduction (\% Bodyweight) & 20.5 & 5.1 & 11.6 & 38.4 & 20.2 & 4.3 & 11.1 & 29.9 \\
\hline Hip Internal Rotation (\% Bodyweight) & 17.03 & 4.2 & 8.8 & 30.1 & 17.5 & 3.9 & 6.8 & 27.9 \\
\hline Hip External Rotation (\% Bodyweight) & 19.1 & 4.6 & 10.1 & 33.5 & 18.1 & 4.4 & 8.5 & 27.2 \\
\hline Static Postural Stability (EO) (N) & 7.9 & 3.5 & 3.8 & 26.2 & 6.5 & 4.6 & 2.9 & 27.7 \\
\hline Static Postural Stability Eyes (EC)(N) & 20.4 & 9.2 & 7.6 & 75.5 & 14.6 & 6.6 & 4.7 & 33.5 \\
\hline Dynamic Postural Stability & 0.342 & 0.04 & 0.24 & 0.472 & 0.35 & 0.03 & 0.287 & 0.419 \\
\hline
\end{tabular}

$\mathrm{EO}=$ eyes open; $\mathrm{EC}=$ eyes closed

*Reported standard deviation of ground reaction forces in Newtons; ** reported as the dynamic postural stability index, a unitless measure.

Table 3: Correlation Matrix- All Subjects

\begin{tabular}{|l|r|r|r|}
\hline & \multicolumn{3}{|c|}{ All Subjects } \\
& \multicolumn{2}{|c|}{ Static Postural Stability Eyes } & Static Postural Stability Eyes \\
Open & $-0.21(0.008)$ & $-0.26(<0.001)$ & $\begin{array}{r}\text { Dynamic Postural } \\
\text { Stability }\end{array}$ \\
\hline Hip Abduction & $-0.23(0.004)$ & $-0.28(<0.001)$ & $-0.10(0.189)$ \\
Hip Adduction & $-0.29(<0.001)$ & $-0.28(<0.001)$ & $-0.09(0.265)$ \\
Hip Internal & $-0.19(0.014)$ & $-0.20(0.012)$ & $-0.07(0.354)$ \\
Rotation & & & $-0.08(0.286)$ \\
Hip External & & & \\
Rotation & & & \\
\hline
\end{tabular}

Table 4: Correlation Matrix- Male Subjects

\begin{tabular}{|c|c|c|c|}
\hline & \multicolumn{3}{|l|}{ Males } \\
\hline & $\begin{array}{c}\text { Static Postural Stability Eyes } \\
\text { Open }\end{array}$ & $\begin{array}{c}\text { Static Postural Stability Eyes } \\
\text { Closed }\end{array}$ & $\begin{array}{l}\text { Dynamic Postural } \\
\text { Stability }\end{array}$ \\
\hline Hip Abduction & $-0.30(0.001)$ & $-0.26(0.005)$ & $-0.16(0.097)$ \\
\hline Hip Adduction & $-0.34(<0.001)$ & $-0.33(<0.001)$ & $-0.10(0.292)$ \\
\hline $\begin{array}{l}\text { Hip Internal } \\
\text { Rotation }\end{array}$ & $-0.29(0.002)$ & $-0.29(0.002)$ & $-0.07(0.442)$ \\
\hline $\begin{array}{l}\text { Hip External } \\
\text { Rotation }\end{array}$ & $-0.21(0.024)$ & $-0.22(0.020)$ & $-0.10(0.310)$ \\
\hline
\end{tabular}

SEBT and Y-Balance Test consist of maintaining stability on a single-leg while performing reaching activities with the opposite leg. ${ }^{20,21}$ These tests do not require a change of base of support and could be described as somewhere in the middle of the continuum between a static task and a dynamic task. These tests require isometric strength in order to maintain stability while holding reaching positions which could explain the correlations Ambegaonkar et al and Wilson et al found. A single-leg landing task requires a change of base of support, what would likely be considered truly "dynamic", thus increasing the difficulty of the task as compared to the SEBT or Y-Balance Test. Due to the increased difficulty of the task, added proprioceptive, vestibular, and somatosensory input and integration would likely be required in order to maintain balance.

Other studies that have utilized a single-leg landing task have found comparable results to this study. Williams et al utilized the DPSI during single-leg landing activities to measure dynamic postural stability. ${ }^{22}$ They compared these balance scores to measures of LE strength and flexibility. Their reported results showed correlations between flexibility and strength measures in the ankle and knee, but no correlation between hip strength and dynamic postural stability. Williams et al, however, only measured hip abduction to represent hip strength ${ }^{22}$ while this study utilized additional measurements including hip internal rotation, external rotation, and adduction.

Results of correlations between isometric hip strength 
Table 5: Correlation Matrix- Female Subjects

\begin{tabular}{|c|c|c|c|}
\hline & \multicolumn{3}{|l|}{ Females } \\
\hline & $\begin{array}{c}\text { Static Postural Stability Eyes } \\
\text { Open }\end{array}$ & $\begin{array}{l}\text { Static Postural Stability Eyes } \\
\text { Closed }\end{array}$ & $\begin{array}{r}\text { Dynamic Postural } \\
\text { Stability }\end{array}$ \\
\hline Hip Abduction & $-0.03(0.848)$ & $-0.23(0.111)$ & $-0.04(0.776)$ \\
\hline Hip Adduction & $-0.03(0.825)$ & $-0.20(0.170)$ & $-0.03(0.812)$ \\
\hline $\begin{array}{l}\text { Hip Internal } \\
\text { Rotation }\end{array}$ & $-0.29(0.039)$ & $-0.28(0.047)$ & $-0.10(0.502)$ \\
\hline $\begin{array}{l}\text { Hip External } \\
\text { Rotation }\end{array}$ & $-0.22(0.120)$ & $-0.31(0.028)$ & $-0.01(0.945)$ \\
\hline
\end{tabular}

and static postural stability have been found in previous studies. Kim and Kim found similar results in their study assessing the relationship between lower extremity strength and range of motion and static postural stability. ${ }^{23}$ Their study only measured hip flexor and extensor strength, however a significant correlation was found between hip flexor strength and postural sway during a standing balance task. ${ }^{23}$ These findings were surprising as it was hypothesized that due to the low physical demands of the static postural stability tests, less demand would be placed on strength and more on proprioceptive, vestibular, and somatosensory information which were not measured in this study. However, it is worth exploring the notion that static tasks may require more isometric strength while dynamic tasks may require more dynamic muscle functions (ie eccentric, concentric) thus explaining the results of this study.

Future studies regarding this topic may present clinical importance for those attempting to improve dynamic or static postural stability in athletes. This study demonstrated weak correlations between hip strength and static postural stability in mainly male athletes. Due to the low correlations and need for more research, particularly interventionbased studies, definitive statements such as "improvements in hip strength will lead to improvements in static postural stability" cannot be made at this time. It is likely that the relationship between hip strength and postural stability is more complicated than the relationship assessed in the current study.

\section{CONCLUSION}

There was no relationship between isometric hip strength and dynamic postural stability; whereas, there were some relationships between the strength measures and static postural stability. These significant, but minimal correlations were observed in more of the comparisons within the male cohort potentially demonstrating a sex difference. Future research on this relationship and the implications on injury prevention should include other measures of muscle performance (isokinetic strength and/or electromyography) and integrate other assessments of neuromuscular control including proprioceptive, vestibular, and somatosensory integration.

\section{CONFLICTS OF INTEREST \\ All Authors have nothing to disclose \\ Submitted: December 18, 2019 CDT, Accepted: July 24, 2020 CDT}




\section{REFERENCES}

1. Hootman JM, Dick R, Agel J. Epidemiology of collegiate injuries for 15 sports: Summary and recommendations for injury prevention initiatives. $J$ Athl Train. 2007;42(2):311-319.

2. Nadler SF, Malanga GA, Feinberg JH, Rubanni M, Moley P, Foye P. Functional performance deficits in athletes with previous lower extremity injury. Clin J Sport Med. 2002;12(2):73-78. doi:10.1097/00042752-2 00203000-00002

3. Alentorn-Geli E, Myer GD, Silvers HJ, et al. Prevention of non-contact anterior cruciate ligament injuries in soccer players. Part 1: Mechanisms of injury and underlying risk factors. Knee Surg Sports Traumatol Arthrosc. 2009;17(7):705-729. doi:10.1007/ $\underline{\text { s00167-009-0813-1 }}$

4. Beynnon BD, Renström PA, Alosa DM, Baumhauer JF, Vacek PM. Ankle ligament injury risk factors: A prospective study of college athletes. J Orthop Res. 2001;19(2):213-220. doi:10.1016/s0736-0266(00)9000 $\underline{4-4}$

5. Grassi A, Alexiou K, Amendola A, et al. Postural stability deficit could predict ankle sprains: A systematic review. Knee Surg Sports Traumatol Arthrosc. 2018;26(10):3140-3155. doi:10.1007/s0016 7-017-4818-X

6. Willems TM, Witvrouw E, Delbaere K, Mahieu N, De Bourdeaudhuij L, De Clercq D. Intrinsic risk factors for inversion ankle sprains in male subjects: A prospective study. Am J Sports Med.

2005;33(3):415-423. doi:10.1177/0363546504268137

7. McGuine TA, Greene JJ, Best T, Leverson G. Balance as a predictor of ankle injuries in high school basketball players. Clin J Sport Med. 2000;10(4):239-244. doi:10.1097/00042752-20001000 0-00003

8. Akuthota V, Ferreiro A, Moore T, Fredericson M. Core stability exercise principles. Curr Sports Med Rep. 2008;7(1):39-44. doi:10.1097/01.csmr.000030866 $\underline{3.13278 .69}$

9. Fredericson M, Moore T. Muscular balance, core stability, and injury prevention for middle- and longdistance runners. Phys Med Rehabil Clin N Am. 2005;16(3):669-689. doi:10.1016/j.pmr.2005.03.001

10. Leetun DT, Ireland ML, Willson JD, Ballantyne BT, Davis IM. Core stability measures as risk factors for lower extremity injury in athletes. Med Sci Sports Exerc. 2004;36(6):926-934. doi:10.1249/01.mss.00001 28145.75199.c3
11. Wilkerson GB, Colston MA. A refined prediction model for core and lower extremity sprains and strains among collegiate football players. J Athl Train. 2015;50(6):643-650. doi:10.4085/1062-6050-50.2.04

12. Willson JD, Dougherty CP, Ireland ML, Davis IM. Core stability and its relationship to lower extremity function and injury. J Am Acad Orthop Surg. 2005;13(5):316-325. doi:10.5435/00124635-20050900 0-00005

13. Sell TC. An examination, correlation, and comparison of static and dynamic measures of postural stability in healthy, physically active adults. Phys Ther Sport. 2012;13(2):80-86. doi:10.1016/i.pts p.2011.06.006

14. Goldie PA, Bach TM, Evans OM. Force platform measures for evaluating postural control: Reliability and validity. Arch Phys Med Rehabil. 1989;70(7):510-517.

\section{Daniels and Worthingham's Muscle Testing} Techniques of Manual Examination [Computer Program]. Version W.B.: Saunders Company; 2002.

16. Sell TC, Tsai YS, Smoliga JM, Myers JB, Lephart SM. Strength, flexibility, and balance characteristics of highly proficient golfers. J Strength Cond Res. 2007;21(4):1166-1171.

17. Shrout PE, Fleiss JL. Intraclass correlations: Uses in assessing rater reliability. Psychol Bull. 1979;86(2):420-428. doi:10.1037/0033-2909.86.2.420

18. Ambegaonkar JP, Mettinger LM, Caswell SV, Burtt A, Cortes N. Relationships between core endurance, hip strength, and balance in collegiate female athletes. Int J Sports Phys Ther. 2014;9(5):604-616.

19. Wilson BR, Robertson KE, Burnham JM, Yonz MC, Ireland ML, Noehren B. The relationship between hip strength and the Y balance test. J Sport Rehabil. 2018;27(5):445-450. doi:10.1123/jsr.2016-0187

20. Kinzey SJ, Armstrong CW. The reliability of the star-excursion test in assessing dynamic balance. $J$ Orthop Sports Phys Ther. 1998;27(5):356-360. doi:10.2 519/iospt.1998.27.5.356

21. Plisky PJ, Gorman PP, Butler RJ, Kiesel KB, Underwood FB, Elkins B. The reliability of an instrumented device for measuring components of the star excursion balance test. N Am J Sports Phys Ther. 2009;4(2):92-99. 
22. Williams VJ, Nagai T, Sell TC, et al. Prediction of dynamic postural stability during single-leg jump landings by ankle and knee flexibility and strength. $J$ Sport Rehabil. 2016;25(3):266-272. doi:10.1123/jsr.20 15-0001
23. Kim S-G, Kim W-S. Effect of ankle range of motion (ROM) and lower-extremity muscle strength on static balance control ability in young adults: A regression analysis. Med Sci Monit. 2018;24:3168-3175. doi:10.12 659/msm.908260 